\title{
Imperfect Channel Knowledge Based Ergodic Channel Capacity of Transmit Beamforming
}

\author{
Du Yang, Lie-Liang Yang and Lajos Hanzo \\ School of ECS, University of Southampton, SO17 1BJ, UK \\ Tel: +44-23-8059 3364; Fax: +44-23-8059 4508 \\ E-mail: dy05r,lly,lh@ecs.soton.ac.uk; http://www-mobile.ecs.soton.ac.uk
}

\begin{abstract}
In this treatise, we have derived the lower ergodic channel capacity bound of a closed-loop transmit beamforming system scheme, when considering the effects of channel estimation errors, transmit beamforming vector quantization errors, the effective capacity loss caused by pilot symbols and that imposed by the channel information feedback bits. We intend to answer the question "whether the potential capacity improvement achieved by using a closed-loop design and an increased number of transmit antennas will be completely eroded by the imperfections of the channel information acquisition and signalling?". Based on our investigations, we found that the capacity improvements predicted are indeed achievable for slowly fading channels, but erode for realtively rapidly fading channels. An improved closedloop transmit beamforming scheme is proposed for rapidly fading channels.
\end{abstract}

Index Terms-Closed-loop transmit beamforming, ergodic capacity, imperfect channel knowledge, pilot-assisted channel estimation

\section{INTRODUCTION}

In a single-user point-to-point communication scenario associated with perfect Channel State Information at both the Receiver (CSIR) and the transmitter (CSIT), the achievable ergodic channel capacity (characterized in terms of bit/s/Hz) can be substantially increased compared to having no CSIT, especially when having more transmit antennas than receive antennas [1], [2]. This is achieved by exploiting the CSIT with the aid of sophisticated transmit pre-processing techniques [3]-[5], However, having perfect CSIT at our disposal is not realistic, especially at high Doppler frequencies. In a Time-Division-Duplex (TDD) system, the CSIT can be obtained with the aid of the received pilots or data symbols by exploiting the similarity of the forward and reverse channels. In a Frequency-Division-Duplex (FDD) system, a widely accepted model is that the CSI is first estimated at the receiver, and then quantized based on a pre-defined codebook known by both transmitter and receiver. The index of the chosen codeword is then fed back to the transmitter via a potentially errorprone channel, and the CSI is reconstructed at the transmitter side. Naturally, the practically attainable CSIT is imperfect. Moreover, the employment of pilot symbols and feedback

\footnotetext{
${ }^{1}$ The work reported in this paper has formed part of the Core 4 Research Programme of the Virtual Center of Excellence in Mobile and Personal Communications, Mobile VCE, www.mobilevce.com, whose funding support, including that of EPSRC, is gratefully acknowledged. Fully detailed technical reports on this research are available to Industrial Members of Mobile VCE.
}

bits imposes a capacity loss. Will the promised ergodic capacity gain completely erode because of the loss caused by imperfect CSIT acquisition and signalling? Does employing more transmit antennas have the potential of improving the effective achievable capacity? These are the questions we intend to answer in this treatise. Four design factors may cause performance degradation, namely the channel estimation error, transmit beamforming vector quantization error, the throughput loss caused by pilot symbols and that imposed by the feedback bits, which are investigated in this study. The effects of channel-induced feedback errors and delays are left for further study.

The analysis of the achievable ergodic channel capacity when using imperfect CSIT is not an entirely new research topic [6]-[9], but it still has open facets. Compared to the previous results in the open literature, the new contributions of our study are as follows. Firstly, we consider realistic pilotassisted channel estimation and quantify the effective capacity loss imposed by pilot signalling. Secondly, we invoked the upper distortion bound of the quantized beamforming vector from lated in [10] to circumvent specific quantization methods, and derive the ergodic channel capacity lower bounds for realistic systems. Moreover, we proposed a modified closedloop TxBF scheme in order to explore the benefits of having multiple transmit antennas, when the channel's coherence time is relatively low.

In Section II, the system model, the pilot-assisted channel estimation algorithm and the derivation of the upper bounds of a beamforming vector quantizer using rate-distortion theory [10] are briefly introduced. Using this knowledge, the ergodic channel capacity of a closed-loop TxBF systems having imperfect CSI is analysed in Section III. Our simulation results and discussions are provided in Section IV. An improved closed-loop TxBF scheme is proposed in Section V. Finally, our conclusion are offered in Section VI.

\section{PRELIMINARIES}

\section{A. System Model and Pilot-assisted Channel Estimation}

A Multiple-Input-Single-Output (MISO) FDD Downlink (DL) system having $N_{t}$ transmit antennas and a single receive antenna is illustrated in Fig. 1. The channel is assumed to be a spatially independent narrow-band slow Rayleigh fading 

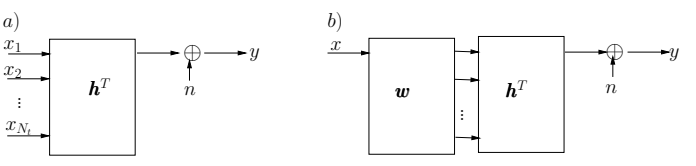

Fig. 1. System model a) open-loop system; b) closed-loop TxBF system.

channel $h \in \mathcal{C}^{N_{t} \times 1}$ plus Gaussian noise $n \sim \mathcal{C}\left(0, N_{0}\right)$. The open-loop system operating without CSIT is represented as $y=\boldsymbol{h}^{T} \boldsymbol{x}+n, \boldsymbol{x} \in \mathcal{C}^{N_{t} \times 1}$. By contrast, the closedloop system employing transmit beamforming [11] (TxBF) is characterized by $y=\boldsymbol{h}^{T} \boldsymbol{w} x+n, \boldsymbol{w} \in \mathcal{C}^{N_{t} \times 1}$, where $\boldsymbol{w}$ is the transmit beamforming vector received from the feedback link. Assuming a unity transmission power, we have $E\left[\|\boldsymbol{x}\|^{2}\right]=$ $1, E\left[|x|^{2}\right]=1$ and $\boldsymbol{w}^{H} \boldsymbol{w}=1$. By setting $\boldsymbol{w}=\frac{\boldsymbol{h}^{*}}{\|\boldsymbol{h}\|}$, the mutual information between the transmitter and the receiver is maximised [8]. The channel can be modelled by block fading. A transmission frame consisting of $K$ symbols is transmitted within the channel's coherence period $T_{c o h}$. The pilot symbols $p_{i j},\left|p_{i j}\right|^{2}=1$ are allocated in the first $L N_{t}$ symbols in a timeorthogonal manner for channel estimation [12] as shown in Fig. 2. Hence, the estimated channel vector obeys $\hat{h}=\boldsymbol{h}+\boldsymbol{h}_{e}$, $\boldsymbol{h}_{e} \sim \mathcal{C N}\left(0, \frac{N_{0}}{L} \boldsymbol{I}_{N_{t}}\right)$.

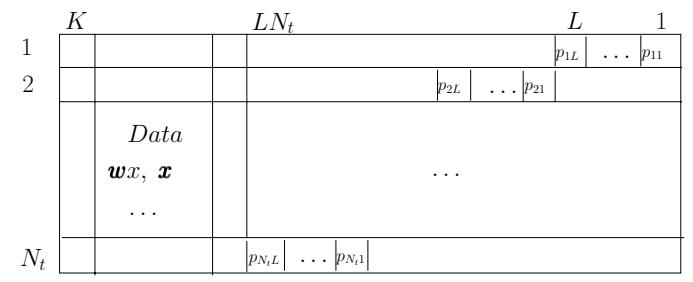

Fig. 2. Transmission frame structure of the $N_{t}$ transmit antennas consisting of $L N_{t}$ pilots and $\left(K-L N_{t}\right)$ data symbols.

\section{B. Upper Distortion Bound of a Beamforming Vector Quan- tizer}

Assuming having perfect CSI at the receiver (CSIR), a quantizer at the receiver takes the CSI $h$ as its input, and selects the beamforming vector $\boldsymbol{w}$ from a pre-defined codebook having the cardinality of $\mathcal{B}$ in order to minimise the resultant distortion. The index of the chosen codeword is then fed back to the transmitter using $R=\log _{2} \mathcal{B}$ bits. It is clear that the performance of a closed-loop TxBF system is affected by several factors, such as the quantization distortion metric employed, the codebook efficiency and the codebook size. We employed the commonly used distortion metric $d_{1}(\boldsymbol{h}, \boldsymbol{w})$ in our study, which is the instantaneous channel gain loss expressed as [10], [11] $d_{1}\left(\boldsymbol{h}, \boldsymbol{w}_{i}\right)=\|\boldsymbol{h}\|^{2}-\left\|\boldsymbol{h}^{T} \boldsymbol{w}_{i}\right\|^{2}, \quad i \in[1, \mathcal{B}]$. Consequently, the codebook is designed in order to minimise the average distortion $D=E_{\boldsymbol{h}}\left[\|\boldsymbol{h}\|^{2}-\left\|\boldsymbol{h}^{T} \boldsymbol{w}\right\|^{2}\right]$. In order to derive a relationship between the feedback bit-rate $R$ and the corresponding distortion $D$, regardless of the specific codebook design method, rate-distortion theory [13] was used in [10]. The analysis provided in [10] shows that using a total of $R$ bits per CIR vector quantization codebook index, the upper bound of the averaged distortions $D_{u}$ is expressed as [10]:

$$
D_{u}(R)=\left(N_{t}-1\right) * 2^{-\frac{R}{N_{t}}}, 0 \leq D_{u} \leq\left(N_{t}-1\right) .
$$

The analysis provided in [10] also shows that using an $R$ bit feedback, the instantaneous distortion $d_{1}(\boldsymbol{h}, \boldsymbol{w} \mid \boldsymbol{h}, R)$ for a given $h$ can be calculated using Monte-Carlo method as

$$
d_{1}(\boldsymbol{h}, \boldsymbol{w} \mid \boldsymbol{h}, R)=\frac{1}{V} \sum_{v=1}^{V}\|\boldsymbol{h}\|^{2}-\frac{\left|\boldsymbol{h}^{H} \boldsymbol{m}_{v}\right|^{2}}{\|\left.\boldsymbol{m}_{v}\right|^{2}}
$$

where the vector $\boldsymbol{m}_{v}$ is a generated complex-valued $N_{t^{-}}$ dimensional random vector having distribution $\boldsymbol{m}_{v} \sim$ $\mathcal{C N}\left(\frac{N_{t}-1-D}{N_{t}-1} \boldsymbol{h}, \quad\left(\frac{N_{t}-1-D}{N_{t}-1}\right)^{2}\left(\frac{D}{N_{t}-1-D}\right) \boldsymbol{I}_{N_{t}}\right)$, and $V$ is a large integer (e.g. $V=10^{4}$ ). Please refer to [10] for more detail concerning the derivation of above equations.

\section{ERgodic Channel CAPACITy AnAlysis}

In practice, the beamforming vector $\boldsymbol{w}$ is selected from a codebook $\mathcal{W}$ having a cardinality of $\mathcal{B}$ based on the estimated CSIR $\hat{h}$, and its index is fed back to the transmitter using $R=\log _{2} \mathcal{B}$ bits every $T_{c o h}$. The capacity losses caused by the pilot signalling and the index feedback are $\frac{L N_{t}}{K}$ and $\frac{R}{K}$, respectively. The upper bound of the channel gain reduction $d_{1}(\hat{\boldsymbol{h}}, \boldsymbol{w} \mid \hat{\boldsymbol{h}}, R)$ due to quantization using $R$ bits for feedback can be derived using Equation (2) by setting $D=D_{u}(R)$. The received signal $y$ will be rewritten as

$$
y=\boldsymbol{h}^{T} \boldsymbol{w} x+n=\hat{\boldsymbol{h}}^{T} \boldsymbol{w} x+n^{\prime}, \quad n^{\prime}=n-\boldsymbol{h}_{e}^{T} \boldsymbol{w} x,
$$

where $n^{\prime}$ is the effective noise as a result of the channel estimation error and the beamforming vector quantization error. Since $\boldsymbol{w}^{H} \boldsymbol{w}=1, \boldsymbol{h}_{e}^{T} \boldsymbol{w} \leq\left\|\boldsymbol{h}_{e}\right\|$, we define $\boldsymbol{h}_{e}^{T} \boldsymbol{w}=\left\|\boldsymbol{h}_{e}\right\| \rho e^{j \varphi}$, where $\rho \in[0,1], \varphi \in[-\pi, \pi]$. For a given $h$, the variance of the equivalent noise $n^{\prime}$ is

$$
\begin{aligned}
E\left(n^{\prime} n^{\prime *} \mid \boldsymbol{h}\right) & =E\left(n n^{*}\right)+E\left(\left(\left\|\boldsymbol{h}_{e}\right\| \rho e^{j \varphi} x \mid \boldsymbol{h}\right)\left(\left\|\boldsymbol{h}_{e}\right\| \rho e^{j \varphi} x\right)^{H} \mid \boldsymbol{h}\right) \\
& =E\left(n n^{*}\right)+E\left(\rho^{2}\left\|\boldsymbol{h}_{e}\right\|^{2} x x^{H} \mid \boldsymbol{h}\right) \\
& =N_{0}+E\left(\rho^{2}\left\|\boldsymbol{h}_{e}\right\|^{2} \mid \boldsymbol{h}\right) .
\end{aligned}
$$

We infer from Equation (4) that $n^{\prime}$ is CIR $\boldsymbol{h}$ dependent and not Gaussian distributed, whose variance can be derived using Monte-Carlo simulations. However, assuming that $n^{\prime}$ is Gaussian distributed, we can derive the lower bound of the ergodic channel capacity in the form of

$$
\begin{aligned}
& C_{\text {closed- }-\hat{\boldsymbol{h}}-\overline{\boldsymbol{h}}-l}= \\
& \frac{K-L N_{t}}{K} E_{\hat{\boldsymbol{h}}}\left(\log _{2}\left(1+\frac{\|\hat{\boldsymbol{h}}\|^{2}-d_{1}(\hat{\boldsymbol{h}}, \boldsymbol{w} \mid \hat{\boldsymbol{h}}, R)}{N_{0}+E\left(\rho^{2}\left\|\boldsymbol{h}_{e}\right\|^{2} \mid \hat{\boldsymbol{h}}\right)}\right)\right)-\frac{R}{K},
\end{aligned}
$$

which combines the effect of the channel estimation error, transmit beamforming vector quantization error, the throughput loss caused by pilot symbols and that imposed by the feedback bits. From Equation (5), it is evident that increasing the value of $K$ will increase the achievable channel capacity 
of a closed-loop TxBF system. However, the other three parameters $L, N_{t}$ and $R$ all have two opposite effects on the achievable capacity. For example, upon increasing $L \rightarrow \infty$, the achievable capacity can be improved by increasing the channel estimation accuracy according to $\frac{N_{0}}{L_{K}} \rightarrow 0$, but imposing an increased capacity loss yielding $\frac{K-L N_{t}}{K} \rightarrow 0$. Similarly, increasing $N_{t}$ according to $N_{t} \rightarrow \infty$ is capable of improving the achievable ergodic capacity by increasing the channel gain $E\left(\|\hat{\boldsymbol{h}}\|^{2}\right) \rightarrow \infty$, but imposes a capacity loss due to the requirement of using more pilot symbols yielding $\frac{K-L N_{t}}{K} \rightarrow 0$. Moreover, a large value of $R \rightarrow \infty$ is capable of improving the achievable capacity by providing more accurate CSIT $d_{1}(\hat{\boldsymbol{h}}, \boldsymbol{w} \mid \hat{\boldsymbol{h}}, R) \rightarrow 0$. However, it will also degrade the achievable capacity, because the feedback overhead obeys $\frac{R}{K} \rightarrow \infty$.

\section{Simulation Results And Discussion}

TABLE I

SiMULATION PARAMETERS

\begin{tabular}{|l|l|}
\hline Number of transmit antenna $N_{t}$ & 2,5 \\
\hline Number of receive antenna $N_{r}$ & 1 \\
\hline SNR in dB & $5 \mathrm{~dB}$ \\
\hline Normalised Doppler Frequency $f_{d m-n o r m}$ & $0.1,0.001$ \\
\hline Number of symbols $K$ per frame during a $T_{c o h}$ & $10,10^{3}$ \\
\hline Pilot overhead $\eta=\frac{L N_{t}}{K}$ & $(0.1 \%, 1 \%, 10 \%) N_{t}$ \\
\hline Number of feedback bits $R$ per CIR for a $T_{c o h}$ & $1 \sim 10$ \\
\hline
\end{tabular}

Using the parameters stated in Table I, the simulation results of Fig. 4 demonstrate that when the channel's Doppler frequency is as low as $f_{d m-n o r m}=0.001$, the potential throughput improvement of using a closed-loop TxBF system is indeed achievable. However, for a channel Doppler frequency as high as $f_{d m-n o r m}=0.1$, the performance of a closed-loop TxBF system becomes even worse than that of its corresponding open-loop counterpart. Moreover, Fig. 4 demonstrate that for slowly fluctuating channels, a moderate increase of $L, R$ and $N_{t}$ is capable of increasing the achievable capacity. By contrast, when the channel's Doppler frequency is as high as $f_{d m-n o r m}=0.1$, employing less pilot symbols $L$, less feedback bits $R$ and less transmit antennas are in fact capable of achieving an increased capacity. In order to identify the dominant factors resulting in a performance degradation for a rapidly fading channel, the achievable ergodic capacity of a closed-loop TxBF system assuming perfect CSIR and quantized CSIT is shown by the line marked by stars in Fig. 4 c), which is significantly higher than the open-loop capacity bound using perfect CSIR. It is demonstrated that the main reason for the poor performance of a closed-loop TxBF system for a channel associated with a low coherence-time is its inability of affording the inclusion of a sufficiently high number of pilot symbols in order to achieve a sufficiently accurate CSIR knowledge, especially when $N_{t}$ is high. Hence, we proposed a modified closed-loop TxBF scheme in Section V.

\section{Modified Closed-Loop TxBF SCHEME For Channels Having a Low COHERENCE-Time}

The proposed new frame structure is shown in Fig. 3. The main difference in comparison to that of Fig. 2 is that the transmitter is configured to subject the pilot symbols to the effects of the legitimate beamforming vectors in the codebook. Accordingly, instead of estimating the true CIR $h$, the receiver simply has to determine which particular $\boldsymbol{w}_{i}$ vector of the codebook would provide the highest receive energy $E\left(|y|^{2}\right)=$ $E\left(\left|\boldsymbol{h}^{T} \boldsymbol{w}_{i}\right|^{2}+|n|^{2}\right)$, and has to inform the transmitter using $R=\log _{2} \mathcal{B}$ bits. As a result, the number of pilot symbols $L$ is independent of the number of transmit antennas $N_{t}$. This will dramatically reduce the capacity loss caused by pilot symbols, especially when the value of $N_{t}$ is high, while $L$ and $\mathcal{B}$ is limited. For example, if we have $K=10, N_{t}=5, L=1, R=$ 1 , using the frame structure of Fig. 2, $50 \%$ of the channel capacity loss is imposed by the insertion of pilot symbols. Using the proposed scheme, only $20 \%$ of the effective capacity is used for channel estimation.

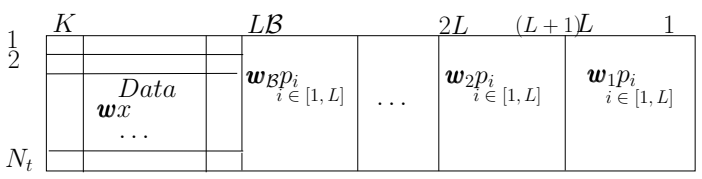

Fig. 3. The proposed frame structure consisting of $L \mathcal{B}$ pilots and $(K-L \mathcal{B})$ data symbols.

Using the proposed scheme obeying the transmission frame structure shown in Fig. 3, the receiver estimates the equivalent channel $h_{i}^{\prime \prime}=\boldsymbol{h}^{T} \boldsymbol{w}_{i}, i \in[1, \mathcal{B}]$ by averaging the $L$ pilots according to $\hat{h}_{i}^{\prime \prime}=h_{i}^{\prime \prime}+\frac{1}{L} \sum_{l=1}^{L} n_{l}=h_{i}^{\prime \prime}+h_{e}^{\prime \prime}$, where $h_{e}^{\prime \prime}$ represents the CIR estimation error, and $h_{e}^{\prime \prime} \sim \mathcal{C N}\left(0, \frac{1}{L} N_{0}\right.$. Assuming that the right codeword is chosen by comparing the energy of the estimated equivalent channel $\boldsymbol{w}=\max _{\boldsymbol{w}_{i}}\left|\hat{h}_{i}^{\prime \prime}\right|^{2}$, which is likely to be the case when the noise variance is low, the lower bound of the ergodic capacity can be formulated as

$$
\frac{K-L * 2^{R}}{K} E_{\boldsymbol{h}}\left(\log _{2}\left(1+\frac{\|\boldsymbol{h}\|^{2}-d_{1}(\boldsymbol{h}, \boldsymbol{w} \mid \boldsymbol{h}, R)}{N_{0}+\frac{N_{0}}{L}}\right)\right)-\frac{R}{K} .
$$

However at low SNRs, it is possible that the chosen codeword is not the optimal one. For this case, we characterise the lower bound of the achievable performance using random beamforming, where a random unitary beamforming vector $w$ is chosen regardless of the current true CIR $\boldsymbol{h}$. Compared to the lower bound of the ergodic capacity expressed in Equation (5) using the frame structure of Fig. 2, there are two major differences. Firstly, the pilot symbol overhead is $\eta=\frac{2^{\mathcal{R}} L}{K}$ instead of $\eta=\frac{L N_{t}}{K}$, which becomes independent of the number of transmit antennas, but it is related to the codebook size $\mathcal{B}$. Secondly, the effective noise is still a Gaussian variable having a variance simply expressed as $N_{0}+\frac{N_{0}}{L}$.

The ergodic capacity of a closed-loop TxBF system having parameters of $N_{t}=2,5 ; K=10 ; L=1 ; R=1$ and using the proposed scheme is shown in Fig. 5. It demonstrate that

1) Assuming the right beamforming vector codeword is chosen from the codebook, the closed-loop system using the proposed scheme outperforms the corresponding 
open-loop system. Moreover, employing more transmit antennas provides a slightly increased capacity. For example, when the number of transmit antennas increases from 2 to 5, the ergodic channel capacity quantified at high SNRs is increased by about $0.5 \mathrm{bits} / \mathrm{s} / \mathrm{Hz}$;

2) If the beamforming vector $\boldsymbol{w}$ is chosen randomly, then the performance of a closed-loop system associated with $N_{t}=2$ transmit antennas becomes similar to that of the corresponding open-loop system. When the number of transmit antennas is increased, the attainable performance becomes almost identical to that shown in Fig. 5.

\section{CONCLUSION}

In this treatise, we have derived the lower ergodic channel capacity bound of a closed-loop TxBF system, when considering the effects of channel estimation errors, tansmit beamforming vector quantization errors, the effective capacity loss caused by pilot symbols and that imposed by the CSI feedback bits in order to answer the questions posed in Section I. Explicitly, the potential capacity improvements of using a closed-loop design and an increased number of transmit antennas are indeed achieveable for slowly fading channels, but erode for relatively rapidly fading channels. For a channel associated with a short coherence time, the modified closed-loop TxBF scheme of Section V is capable of providing a capacity improvement than the corresponding open-loop system associated with the same number of transmit antennas.

\section{REFERENCES}

[1] M. Vu and A. Paulraj, "MIMO wireless linear precoding," IEEE Signal Processing Magazine, vol. 24, no. 5, pp. 86-105, Sept. 2007.

[2] L. Yang, Multicarrier Communications. John Wiley \& Sons, 2009.

[3] M. Joham, M. Utschick, and J. A. Nossek, "Linear transmit processing in MIMO communications systems," IEEE Transaction on Signal Processing, vol. 53, no. 8, pp. 2700-2712, 2005.

[4] C. B. Peel, B. M. Hochwald, and A. L. Swindlehurst, "A vector-perturbation technique for near-capacity multiantenna multiuser communication-part I: channel inversion and regularization," IEEE Transactions on Communications, vol. 53, no. 1, pp. 195-202, Jan. 2005.

[5] B. M. Hochwald, C. B. Peel, and A. L. Swindlehurst, "A vector-perturbation technique for near-capacity multiantenna multiuser communication-part II: perturbation," IEEE Transactions on Communications, vol. 53, no. 3, pp. 537-544, Mar. 2005.

[6] A. D. Dabbagh and D. J. Love, "Feedback rate-capacity loss tradeoff for limited feedback MIMO systems," IEEE Transactions on Information Theory, vol. 52, no. 5, pp. 2190-2202, May 2006.

[7] T. Yoo, E. Yoon, and A. Goldsmith, "MIMO capacity with channel uncertainty: does feedback help?" in Global Telecommunications Conference, 2004. GLOBECOM '04. IEEE, vol. 1, Nov./Dec. 2004, pp. 96100.

[8] T. Yoo and A. Goldsmith, "Capacity and power allocation for fading MIMO channels with channel estimation error," IEEE Transactions on Information Theory, vol. 52, no. 5, pp. 2203-2214, May 2006.

[9] J. Zheng and B. D. Rao, "Capacity analysis of MIMO systems using limited feedback transmit precoding schemes," IEEE Transactions on Signal Processing, vol. 56, pp. 2886-2901, July 2008.

[10] P. Xia and G. B. Giannakis, "Design and analysis of transmitbeamforming based on limited-rate feedback," IEEE Transactions on Signal Processing, vol. 54, no. 5, pp. 1853-1863, May 2006.

[11] D. J. Love, R. W. Heath, and T. Strohmer, "Grassmannian beamforming for multiple-input multiple-output wireless systems," IEEE Transactions on Information Theory, vol. 49, no. 10, pp. 2735-2747, Oct. 2003.
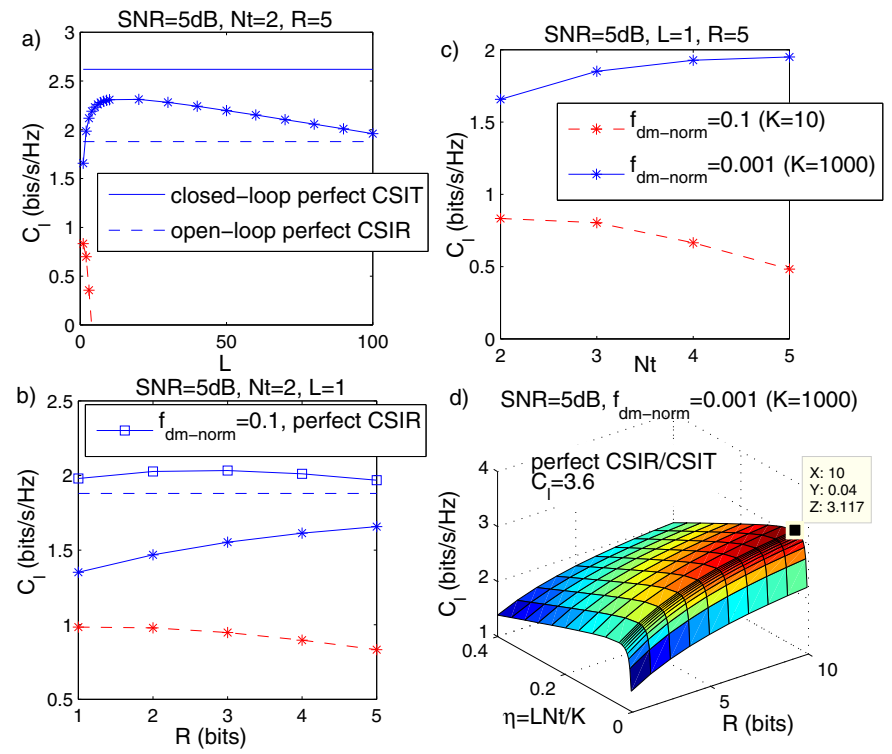

Fig. 4. The lower bound of the achievable ergodic capacity of a closed-loop TxBF system in conjuction with estimated and quantized CSIT $C_{\text {closed }-\hat{\boldsymbol{h}}-\overline{\boldsymbol{h}}-l}$ versus a) the number of pilot symbols per channel's coherence time $L$, when we have $N_{t}=2 ; R=5$; versus b)the number of feedback bits $R$, when we have $N_{t}=2 ; L=1$; versus c) the number of transmit antennas $N_{t}$, when we have $L=1 ; R=5$; and versus d)the number of feedback bits $R$ and pilot overhead $\eta$, when we have $N_{t}=4$. The SNR is assumed to be $5 \mathrm{~dB}$, and the normalised Doppler frequency is assumed to be $f_{d m-n o r m}=0.1 / 0.001(K=10 / 1000)$, respectively. The results were plotted from Equation (5).

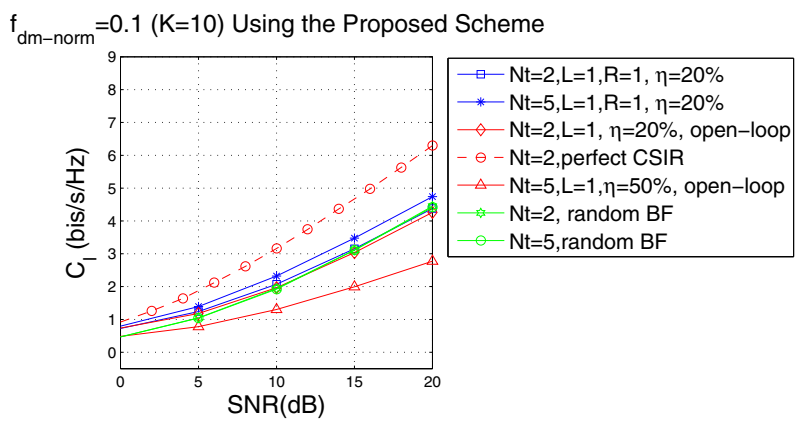

Fig. 5. The lower bound of the ergodic channel capacity of a closed-loop TxBF system in conjunction with estimated and quantized CSIT using the proposed frame structure in Fig. 3 namely $C_{\text {closed }}$ new - scheme $-l$ versus SNR, when we have $N_{t}=2,5 ; f_{\text {dm-norm }}=0.1(K=10) ; L=1 ; R=1,5$, respectively. The results were plotted from Equation (6). Ergodic channel capacity for $N_{t}=2,5, S N R=5 d B$, when assuming perfect CSIR and CSIT calculated from $C_{\text {open }}=E_{\boldsymbol{h}}\left(1+\frac{\left.\|\boldsymbol{h}\|\right|^{2}}{N_{0} N_{t}}\right)$ are also plotted for comparison .

[12] D. Samardzija and N. Mandayam, "Pilot-assisted estimation of MIMO fading channel response and achievable data rates," IEEE Transactions on Signal Processing, vol. 51, no. 11, pp. 2882-2890, Nov. 2003.

[13] J. Proakis, Digital Communications. McGraw-Hill, 1995. 